\title{
Developments in tracking with STAR's heavy flavor tracker
}

\author{
Gene Van Buren* \\ Brookhaven National Laboratory \\ E-mail: geneabnl.gov \\ L. Didenko, Y. Fisyak, L. Hajdu, J. Lauret, V. Perevotzchikov, D. Smirnov, \\ F. Videbaek, J. Webb \\ Brookhaven National Laboratory
}

\section{Dong, M. Mustafa, H. Qiu, A. Schmah}

Lawrence Berkeley National Laboratory

\section{J. Bouchet, M. Lomnitz}

Kent State University

\section{Y. Wang}

Central China Normal University

\section{Z. Ye}

University of Illinois at Chicago

\section{for the STAR Collaboration}

\begin{abstract}
A primary goal of the high luminosity era at RHIC will be the study of heavy quark behavior in Quark Gluon Plasma. The integration of high precision silicon-based tracking in the form of the Heavy Flavor Tracker for the STAR Experiment should enable the reconstruction and identification of charmed hadron decays, working in concert with STAR's Time Projection Chamber to determine momenta and displacement of decay daughters from the primary collision vertex. To reach the precision demands, the new detectors must be calibrated and sufficiently accounted in tracking to observe charmed hadrons with high signal-to-noise. In this paper we will review the STAR Collaboration's developments and achievements in this critical effort.
\end{abstract}

24th International Workshop on Vertex Detector -VERTEX2015-

1-5 June 2015

Santa Fe, New Mexico, USA

* Speaker. 


\section{Introduction}

One of the most intriguing open questions in nuclear physics today is whether the medium created in high-energy heavy-ion collisions interacts with heavy quarks any differently than with light quarks. Toward answering that question, experiments like the Solenoidal Tracker at RHIC (STAR) [1] are interested in identifying produced open-charm hadrons, whose decay lengths are too short to instrumentally directly detect. Instead, decay products are used to reconstruct the parent hadron, such as $D^{0} \rightarrow K^{-} \pi^{+}$. However, in a high-multiplicity heavy-ion collision environment, the combinatorial background with this method dwarfs the signal, unless some means of distinguishing secondary decay daughters from primary collision products can be applied. Simpler analyses, such as determining yields, have been successful in STAR without such discrimination despite the low signal-to-noise [2], but correlation analyses like flow demand it. This defines the requirement for precision tracking with devices like the STAR Heavy Flavor Tracker (HFT) [3], and represents the goal of the effort we present here.

Mid-rapidity tracking at STAR has historically involved two primary components: a $0.5 \mathrm{~T}$ solenoidal magnet [4], enclosing a large Time Projection Chamber (TPC) [5]. These remain the critical means for measuring particle momenta, but projection resolutions from the inner active TPC radius of $\sim 60 \mathrm{~cm}$ to the primary vertex (distance of closest approach, or DCA) of 2-3 mm are not good enough to meet the aforementioned requirement. Tracking is further aided by three inner elements that comprise the HFT: (1) SSD: fast, double-sided (stereoscopic) silicon strips at radii of $\sim 22 \mathrm{~cm}$ (SSD hit usage is not presented here) [6]; (2) IST: fast, single-sided silicon pads at radii of $\sim 14 \mathrm{~cm}$; and (3) PXL: $20.7 \times 20.7 \mu \mathrm{m}$ Monolithic Active Pixel Sensors (MAPS) at radii of $\sim 8 \mathrm{~cm}$ and $\sim 2.8 \mathrm{~cm}$ [7]. Limitations in this setup include lengthy integration windows for the TPC ( 400 collider bunch crossings) and the PXL ( 1750 crossings).

\section{Alignment Calibrations}

Precision tracking requires precision calibration, and for this effort the most crucial was the alignment of the HFT components. Survey measurements provided the starting point for alignments. PXL sensor surfaces underwent both visual and stylus scans on-sector. Deviations from planarity were fit using thin plate splines, and were of the order of a few tens of microns. Although these deviations are a little larger than the in-plane hit reconstruction errors, the radial nature of the tracks would have resulted in much smaller errors when deviations were projected to the plane. The deviations were accounted nevertheless in the reconstructed hit positions.

Surveys were also used to fix the PXL sensor-to-ladder and ladder-to-sector alignments, but further alignments utilized cosmic rays, taken without a magnetic field, which crossed through the middle of the HFT. From these, residuals to straight-line "tracklet" fits could be iteratively minimized. This method was first used to coarsely align two semi-cylindrical PXL halves to each other, then more finely for the individual sectors. To avoid using poorly reconstructed hits, sectors were aligned with respect to only a few sectors on the opposite side. A single PXL sector served as a fixed reference. Residuals from before and after the half-to-half alignment of the PXL can be seen in Fig. 1. Final achieved position uncertainties were in the range of $\sim 10-20 \mu \mathrm{m}$ for the inner PXL layer, and $\sim 15-30 \mu \mathrm{m}$ for the outer PXL. The same technique was used to align the whole IST 

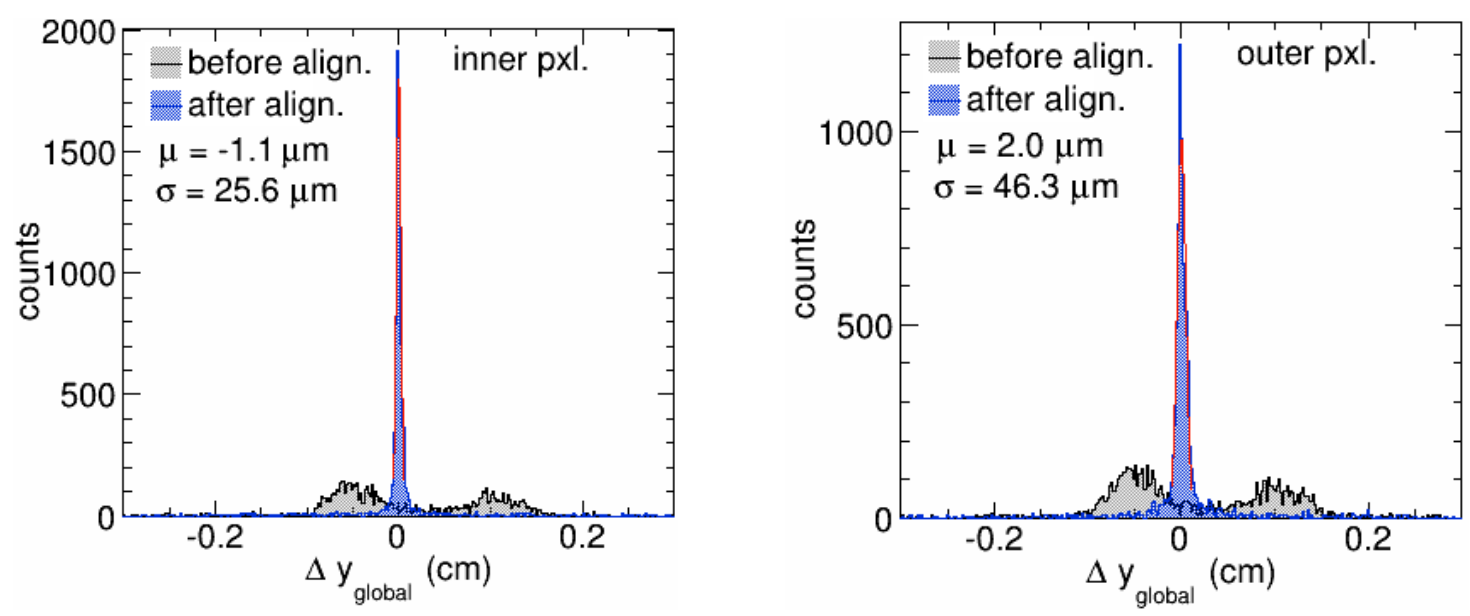

Figure 1: (color online) Examples of residuals in the inner (left) and outer (right) PXL layers before (gray shading) and after (blue shading) the PXL half-to-half internal alignment. Parameters of Gaussian fits (red curves) to the after-alignment distributions are shown; final residuals improved further upon these values.

to the whole PXL, but an attempt to align individual IST ladders showed no notable improvement over survey, so the survey was retained. Final IST position uncertainties were $\sim 200 \times \sim 1800 \mu \mathrm{m}$ in $r \phi \times z$, consistent with expectations based on the pad size [8]. These HFT alignments were checked for stability using data samples acquired at the beginning, middle, and end of the 2014 run of the experiment, and demonstrated consistency.

TPC tracking performance is significantly boosted by operating in the full strength STAR magnetic field. Further, a magnetic field allows selection of tracks to exclude those at low momentum susceptible to significant multiple coulomb scattering. These features improve the precision of track projections across the gap from the TPC to the IST, so cosmic rays taken with both field polarities were used in the HFT-to-TPC alignment. However, this calibration strategy obviated interdependence between the HFT-to-TPC rotation, the internal TPC super-sector rotations, and TPC field distortions. Degeneracies remained between the latter two when the TPC was self-calibrated. Two actions were taken to resolve the matter: (1) the TPC distortion and alignment calibrations were iterated, and (2) the final TPC super-sector alignments were determined by the HFT. The HFT was also instrumental in fine tuning the TPC T0 and drift velocities.

\section{Tracking Developments}

STAR track reconstruction for final physics has historically focused predominantly on transverse physics, using simplified versions of the detector geometries to attain reasonably quick reconstruction times given preciously finite CPU resources. Shortcomings in accuracy in the existing tracker have been sufficient for the $\sim 1 \mathrm{~mm}$ scale precision that the TPC can provide in mid-rapidity tracking, and a fundamental limitation against forward tracking in this software has been acceptable $^{1}$. It was understood coming into the HFT era that significant work would be needed to advance performance of the existing tracker to the requisite sub-hundred-microns scale of interest without sacrificing much speed.

\footnotetext{
${ }^{1}$ Tracking in the STAR Forward Time Projection Chambers used an even simpler tool [9].
} 
Another effort began a few years prior intending to revamp the tracking as part of a larger project extending into simulations, with goals of unifying geometries in simulation and reconstruction (including a single abstract interface to GEANT and ROOT/TGeo [10]), using standard energy loss accounting (GEANT propagator), and implementing agnosticism to track orientation (e.g. forward detectors, or far-from-orthogonality). The status of this effort at the critical decision point in the tracking development included some concerns: significant iterative work remained to get the reconstruction performance in pure simulations to the required precision; and the new tracker was nearly twice as slow on tracking with the TPC alone as the existing tracker. Weighing the known work for the existing tracker against the unknown work for the new tracker, and consideration that comparability with past results was clearer using the existing tracker, led us to concentrate for the time being on updating what was already working well.

\subsection{Assessment Tools}

Validation of the simplified detector geometries used in the tracking followed two prongs. First, because a mature tool to convert GEANT into TGeo volumes for STAR was already certified, TGeoChecker proved valuable in finding issues with GEANT volumes as they were coded, such as undeclared overlaps, and illegal extrusions. Next, a tool was developed in-house to compare (in simulation) energy losses in tracking in physical space with GEANT data on energy losses. The latter proved useful in spotting and isolating discrepancies via their physical location.

Automated tests of development software libraries, in which a variety of existing datasets were processed using the latest codes, were performed nightly. Statistics of several reconstruction quantities (e.g. total number of tracks, number of primary tracks, number of primary vertices, reconstruction time per event, etc.) were recorded in database tables. This assisted monitoring code modifications for any unintended side effects with respect to either basic reconstruction performance, or speed performance.

Detailed quality assurance of the performance made use of track-by-track comparisons between simulated tracks and their reconstructed equivalents. This was an extension of an in-house tool to assess deviations in reconstructed track parameters (e.g. pseudorapidity $\eta$ and curvature $q / p_{T}$, where $q$ is the track charge and $p_{T}$ is the transverse momentum), projections (e.g. transverse DCA), and efficiencies. Each of these was examined differentially with respect to numerous variables, such as $p_{T}, \eta$, azimuth $\phi$, and number of fit points. Fig. 2 demonstrates a progression of the transverse DCA performance versus $p_{T}$ using this tool (shown tracks used only the hits available in the TPC, excluding HFT hits). This particular metric was critical in achieving a sufficient representation of the detector geometry in tracking to perform comparably to the pre-HFT state.

\subsection{Geometries in Tracking}

The GEANT geometry representation of STAR is rich in detail (as it should be), with many thousands of intricate little, oddly-oriented and -shaped volumes and sub-volumes. The vast majority of these are support structures, and much of it is unimportant for tracking (e.g. outside the volumes in which tracking is conducted). With the simplified geometries of STAR's existing tracker, even some minute details of volumes within the tracking region are ignorable. Thus the tracker implements only two basic volume types to keep its calculations simple and quick. These 

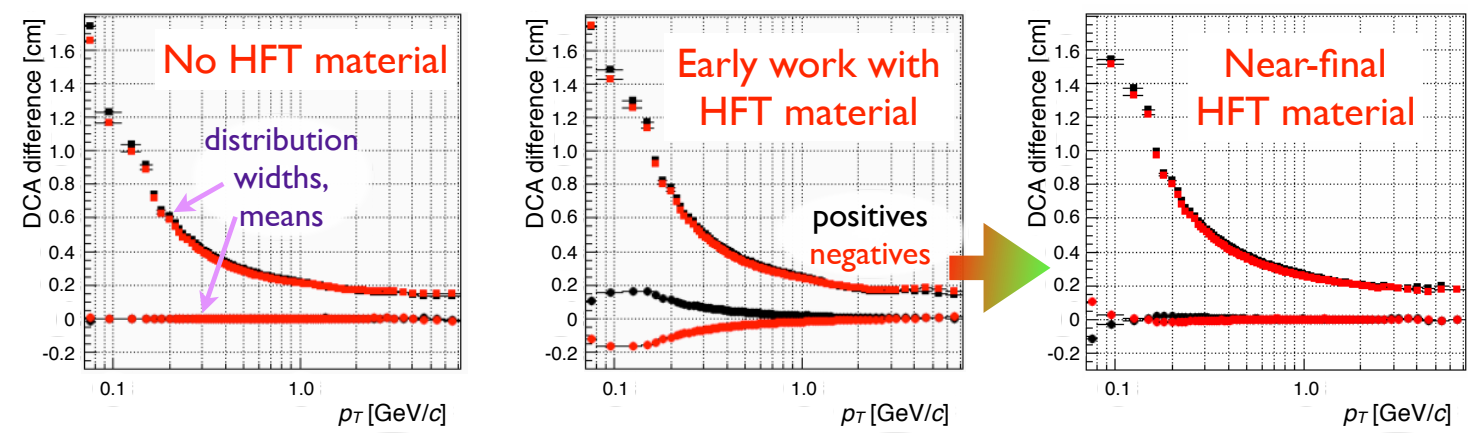

Figure 2: (color online) Progression of the performance of track transverse DCA to primary vertex in $\mathrm{Au}+\mathrm{Au}$ $\sqrt{s_{N N}}=200 \mathrm{GeV}$ simulations. Shown are the widths (squares; some low $p_{T}$ points are off-scale) and means (circles) of the distributions for the difference in transverse DCA between simulation and reconstruction for positively (black) and negatively (red) charged tracks. HFT hits are not used in the tracking here.

are (1) planes (with depth, e.g. boxes), oriented such that one axis is parallel to the experiment's beamline axis, $z$; and (2) cylinders (and cylindrical sections, which may only span a limited range in $\phi$ ) about the $z$ axis. Introducing any other possible volume types or orientations would require significant re-writes of the software, and were deemed too time-consuming for this effort. This means that only limited misalignments of volumes are allowed.

Three methods were employed to represent the STAR geometry model to the tracker. These were: (1) one-to-one copies of compatible volumes from the simulation geometry model; (2) automated averaging of incompatibly-shaped or composite structures (i.e. volumes with several subvolumes inside); and (3) user-supplied implementations of the geometry model using the native tracker description where automated translation was shown to be insufficient. With a great variety in physical HFT pieces, all of these approaches were put to use. It is worth noting that even though the inclusion of SSD hits was not a part of this effort, the geometry of the SSD still needed to be well-represented as the materials were present when the data was acquired.

The tracker scans material volumes across $\phi$ and $z$, within radial layers, for possible intersections with each propagating track as it reaches that radius. It was realized early on that the track projection error should not be accounted in determining whether an intersection occurs with an inactive (no hits) material or not: doing so can hurt (false intersections) as much as it helps (true intersections which otherwise would have been missed).

Intersections are ascertained using a volume's mid-section, defined here as the transverse area at the radius of the volume's center. A mid-section is illustrated on the left side of Fig. 3 for a volume whose depth (radial extent) is notably larger than its mid-section dimensions, which we term as radially-oriented. This tactic is fundamentally quick, but severely limits track orientations for successful determination. Some compensation can be had by assuming any intersecting track traverses the entire volume radially (with a geometrical correction for the angle of intersection), which results in the average over all tracks seeing the expected total amount of material. However, track intersections are all-or-nothing, such that any individual track may see far too little, or far too much material, as exemplified in the middle graphic of Fig. 3. This is particularly unhelpful when the key goal depends on individual track projections. In order not to sacrifice speed, this method was not altered, but radially-oriented plane geometries were: automatic volume segmentation was 


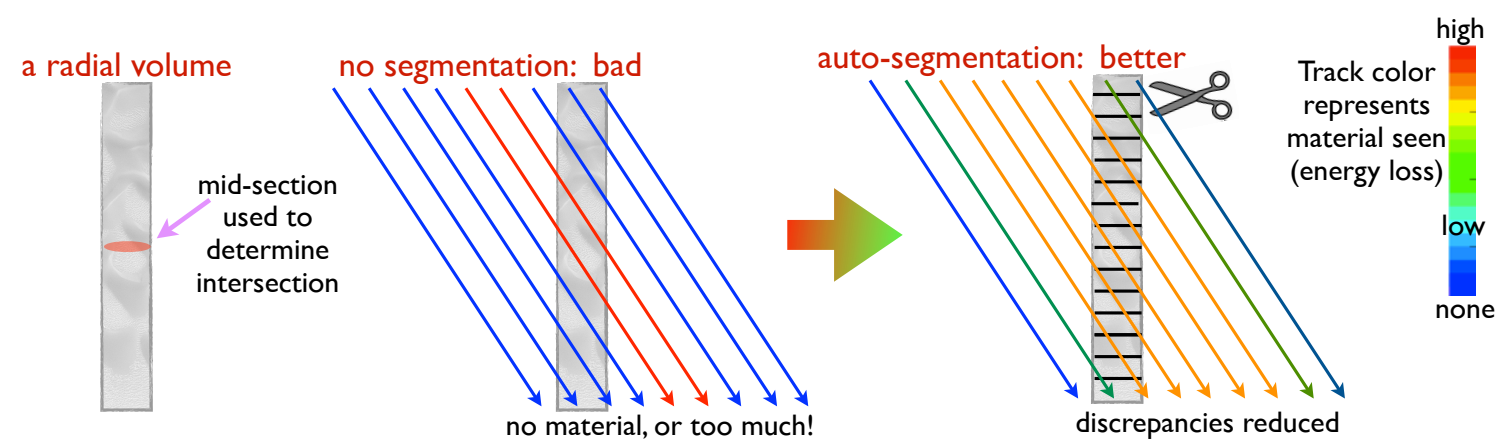

Figure 3: (color online) Sketch of the auto-segmentation feature and its need and benefit. Thin, colored lines represent some possible track trajectories and the amount of material they "see" as they pass the volume. See the text for more details.

implemented to subdivide the volume into multiple radial layers, to the condition where each volume segment is no longer radially-oriented. The right graphic of Fig. 3 shows how each track now sees a more appropriate amount of material, enabling more reasonable energy loss corrections.

A few other important limitations were found and handled. Overlapping tilted layers (e.g. the ladders on some barrel silicon trackers) benefited from being split into two, such that a track can see them as two different radial layers (and find two hits at overlaps) and pass through them in the proper order. Misalignments (non-ideality) in realistic geometries could also cause out-ofradial-order intersections, resulting in propagating tracks in the wrong direction at times. Defining radial layers with a finite radial size (tolerance) alleviated this, after some tuning of the tolerances. Lastly, to ensure the benefits HFT hits provide, priority needed to be given in the intersecting volume search to active volumes where hits may be found.

\subsection{Handling HFT Hits}

With the intended physics analyses emphasizing tracks with HFT hits, less neutrality was granted to finding and assigning them. Consideration of this revealed several more opportunities to improve the software, as follows.

Given the limitations in treating misalignments in the geometry, and the criticality of having HFT hits at their properly (mis)aligned place, it was vital not to mandate hit positions be precisely on the active material with which they were associated, i.e. hits may be located outside their volumes. This was already allowed for the TPC, where distortions and misalignments mandated it, and it became clear that the HFT hits needed this too.

The PXL and TPC are both slow detectors, acquiring a multitude of non-triggered collisions ("pile-up") within the data for a single triggered event. This raises the opportunity for tracks to have a mixture of trigger and pile-up hits. Aside from not wanting such tracks in analyses, it's also vital that these mixed-trigger tracks not rob the proper tracks of their HFT hits. This was achieved by permitting re-use of HFT hits (on up to 5 tracks), which was demonstrated to do more good than harm. In the $2014 \mathrm{Au}+\mathrm{Au}$ data, maximum occupancy in the HFT was under $0.1 \%$ for the innermost PXL layer, so the issue that this resolved was due more to the pile-up in the TPC than in the PXL.

Finally, it was observed in simulation that not finding hits on expected HFT layers was a strong indicator of other HFT hits on the track being incorrectly assigned. A requirement was thus 


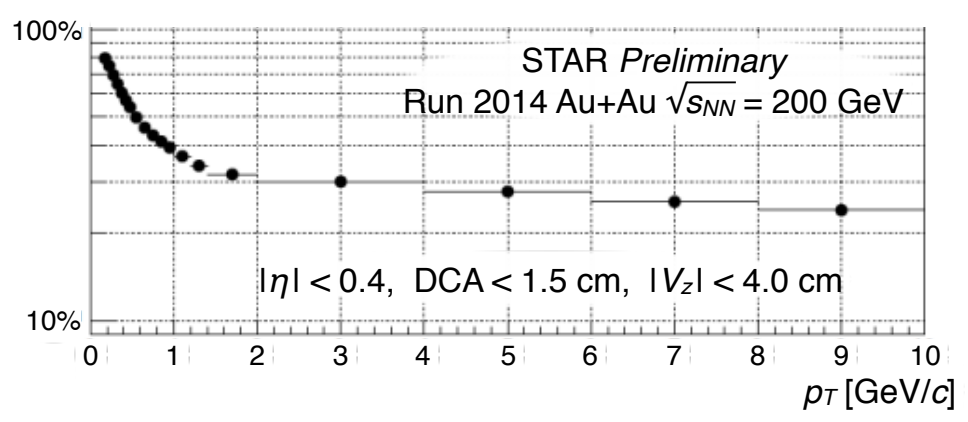

Figure 4: Probability that a track has HFT hits in real STAR data as a function of $p_{T}$, restricted to tracks which project close to a good primary vertex $(\mathrm{DCA}<1.5 \mathrm{~cm})$ and sit well-centered within the acceptance of the active HFT regions $\left(|\eta|<0.4\right.$, and primary vertex position along the $z$ axis $\left.\left|V_{z}\right|<4.0 \mathrm{~cm}\right)$. Simulations with pile-up explained the low $p_{T}$ increase as due to larger track projection errors and associated tolerances for mismatches. Some of the inefficiency is due to dead channels during data-taking.

imposed that there must be hits in each active HFT layer (IST and each pixel layer), otherwise all HFT hits were removed before the final refit, and the track treated as a TPC-only track.

Combining all of these efforts has led to the probability that a track has HFT hits (when it should have them) in real data as seen in Fig. 4. Beyond this positive outcome, pure simulations give hope that there remains potential for improving efficiency of picking up HFT hits even further with future work.

\section{Conclusions}

Ultimately, as evidenced in Fig. 5 where the reconstructed $D^{0}+\overline{D^{0}}$ invariant mass is presented for real heavy-ion collision data with and without, tracking with the HFT is working extremely well toward the goal of reconstructing heavy flavor decays topologically with high signal-to-noise. The 125 million minimum bias $\mathrm{Au}+\mathrm{Au} \sqrt{S_{N N}}=200 \mathrm{GeV}$ events processed for the plot are just a fraction of the $\sim 1.2$ billion recorded on tape, along with another $\sim 1.6$ billion $\mathrm{p}+\mathrm{p}$ events and additional p+Au and p+Al data acquired in 2015, all with $\sqrt{s_{N N}}=200 \mathrm{GeV}$. For the 2014 data, we achieved excellent internal alignment of the HFT subsystems, and the global alignment between HFT and TPC yielded a constructive improvement to the TPC's internal alignment and distortion calibrations. Sufficient material accounting and impressive HFT track reconstruction have been established with updates to STAR's existing tracker and its simplified geometries, while adding a mere $\sim 10 \%$ net to the overall time required to run the whole reconstruction chain. Energy loss in HFT materials has been sufficiently well accounted, and some optimization has been carried out for the use of HFT hits on tracks. Physics analyses from the 2014 data are in progress as of this writing, and the goal of studying the behavior and interactions of heavy flavor produced in high-energy nuclear collisions is in good shape in STAR.

\section{Acknowledgements}

We thank the RACF at BNL and NERSC at LBNL for their support. This work was supported in part by the Offices of NP and HEP within the U.S. DOE Office of Science. 


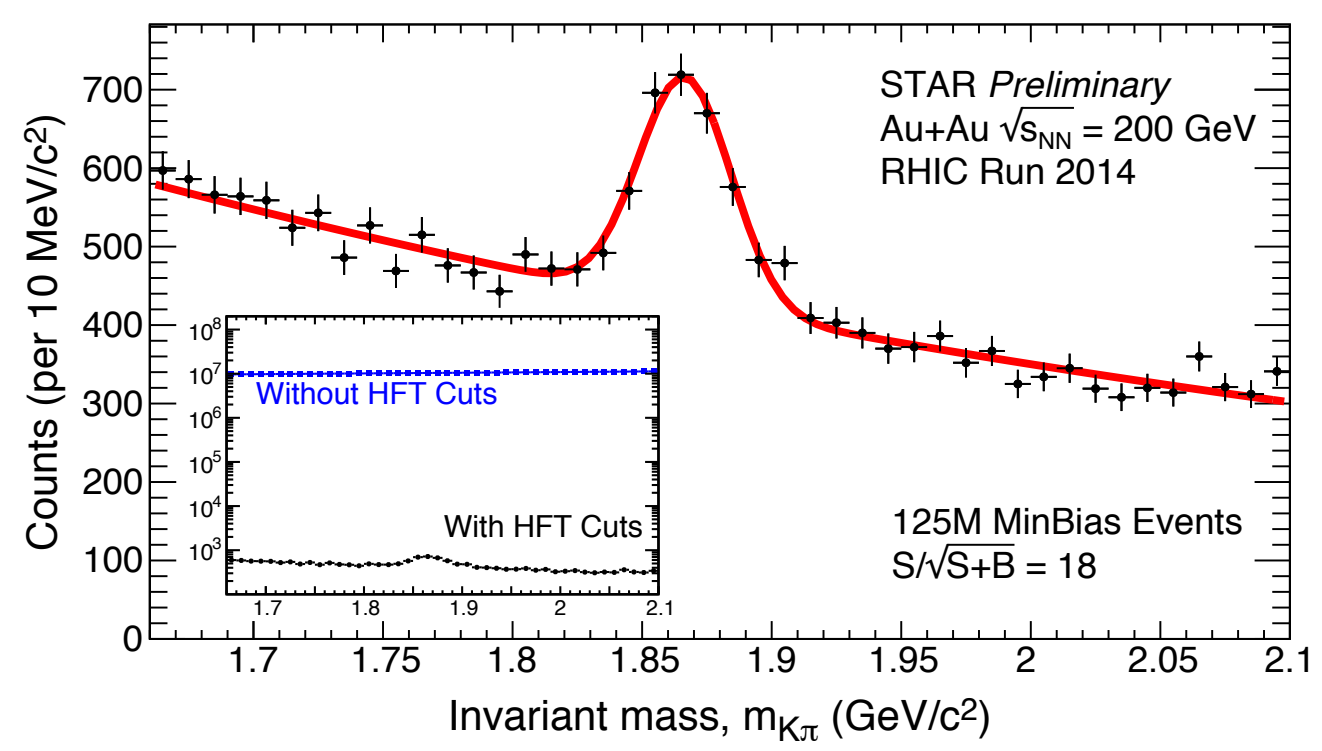

Figure 5: $D^{0}+\overline{D^{0}}$ invariant mass signal in real STAR data with a high signal-to-noise (as measured by signal over the square root of signal+background, $\mathrm{S} / \sqrt{\mathrm{S}+\mathrm{B}}$ ). The inset demonstrates the enormous backgroundreduction power of topological cuts enabled with the HFT tracking.

\section{References}

[1] K.H. Ackermann et al., Nucl. Instr. and Meth. A 499 (2003) 624.

[2] L. Adamczyk et al., Phys. Rev. D 86 (2012) 72013; L. Adamczyk et al., Phys. Rev. Lett. 113 (2014) 142301.

[3] E. Anderssen et al., The STAR Heavy Flavor Tracker, https://drupal.star.bnl.gov/STAR/starnotes/public/sn0600 .

[4] F. Bergsma et al., Nucl. Instr. and Meth. A 499 (2003) 633.

[5] M. Anderson et al., Nucl. Instr. and Meth. A 499 (2003) 699.

[6] L. Arnold et al., Nucl. Instr. and Meth. A 499 (2003) 652.

[7] L. Greiner et al., Nucl. Instr. and Meth. A 650 (2011) 68.

[8] H. Qiu et al., Nucl. Phys. A 931 (2014) 1141.

[9] K.H. Ackermann et al., Nucl. Instr. and Meth. A 499 (2003) 713.

[10] J. Webb, J. Lauret, and V. Perevoztchikov, J. Phys. Conf. Ser. 396 (2012) 022058. 